\title{
El conocimiento en el sistema de salud.
}

\author{
Knowledge in the health system.
}

Jorge Cañarte-Alcívar ${ }^{1}$, Cristhian Marín-Tello. ${ }^{2}$, Leiver Rivera-Chavez. ${ }^{3}$, Pedro FernandezSanchez. ${ }^{4} \&$ Ruth Huerta Vicuña. ${ }^{5}$

Recibido: 10-02-2019 / Revisado: 18-02-209 /Aceptado: 24-03-2019/ Publicado: 05-04-2019

\begin{abstract}
.
DOI: https://doi.org/10.33262/cienciadigital.v3i2.447

Knowledge as a fundamental and intangible element of organized systems aims to maintain the product obtained from the scientific process of its conception, based on the need to transfer it to new actors in that system. Since its inception, knowledge has had different concepts and perceptions and nowadays it is analyzed the importance that it has, by having the quality that it gives from actions to turn decisions, whose organizational characteristics are reflected in the expression of intelligence, efficiency and efficiency stimulating innovation, maintaining the complexity, flexibility and creativity of research products. Health knowledge management uses and creates knowledge based on a dynamic aimed at solving health problems, taking advantage of collective intelligence and contributing to it in a global flow of knowledge related to health.
\end{abstract}

Keywords: System Knowledge, Health Systems, Organized Systems, Health

\section{Resumen.}

El conocimiento como elemento fundamental e intangible de los sistemas organizados tiene como objetivo mantener el producto obtenido del proceso científico de su

${ }^{1}$ Doctor en Medicina y Cirugía, Master en Investigación Clínica y Epidemiológica, Doctor@ en Salud Publica, Docente Investigador, Universidad Técnica de Manabí - jcanarte@utm.edu.ec

${ }^{2}$ Doctor en Medicina y Cirugía, Magister en Gerencia de la Salud, Director Médico Fundación Cottolengo Manta cristhianoamlt@gmail.com

${ }^{3}$ Médico Cirujano, Medico General en Funciones Hospitalaria Hospital General IESS Manta leiver.river@gmail.com

${ }^{4}$ Médico Cirujano, Medico General en Funciones Hospitalaria - Hospital General Dr. Napoleón Dávila Córdova pedrofernandez84@hotmail.com

${ }^{5}$ Médico Cirujano, Medico General en Funciones Hospitalaria Hospital General IESS Manta elissahuerta_@hotmail.com 
concepción, basados en la necesidad de transferir el mismo hacia nuevos actores de dicho sistema. Desde sus inicios el conocimiento ha tenido diversos conceptos y percepciones y en la actualidad se analiza la importancia que este tiene, al tener la cualidad que dé a partir de acciones tornar las decisiones, cuyas características organizacionales se reflejan en la expresión de la inteligencia, eficiencia y eficacia estimulando la innovación, manteniendo la complejidad, flexibilidad y creatividad de los productos de la investigación. La gestión del conocimiento en salud usa y crea conocimientos basados en una dinámica orientada a la solución de problemas de salud aprovechando la inteligencia colectiva y aportando a ella en un flujo global de conocimientos relacionado a la salud.

Palabras claves: Conocimiento Sistema, Sistemas de Salud, Sistemas Organizados, Salud

\section{Introducción.}

\section{Concepción del conocimiento}

Según el diccionario de la Real academia de la lengua española "el conocimiento" es la acción y efecto de conocer; Conocer.(Del lat. cognoscěre).1. tr. Averiguar por el ejercicio de las facultades intelectuales, la naturaleza, cualidades y relaciones de las cosas. Para el diccionario de Oxford el Conocimiento es la comprensión alcanzada a través de la experiencia, la observación o el estudio.

El conocimiento es la manifestación abstracta de experiencias, en donde se conoce y despeja las dudas e interrogantes que se presentan, siendo su único objetivo alimentar a las ciencias fomentando la transferencia del mismo. Para Knowledge O (1) "Intentar comprender la naturaleza del conocimiento ha sido un tema principal de la investigación filosófica durante miles de años" a ser un recurso con enorme potencial impulsar procesos en donde el conocimiento es parte fundamental en los avances intangibles de la sociedad.

Desde Platón (429 -347 aC) con su corriente idealista en donde "las ideas y el pensamiento estaba sobre la realidad y la experiencia" pensamientos eran influenciados por el idealismo para poder plasmarse en la realidad. Platón influenciado por Sócrates (470 - 399 aC) corrobora su posición de que la concepción del conocimiento tenía que ser infalible enmarcando la verdad real (2)

El conocimiento se basaba en la autoridad del filósofo Aristóteles y en la de la Biblia. La ciencia moderna se hizo posible sólo cuando esta autoridad fue desafiada con una llamada a la experiencia por precursores de la nueva ciencia como Galileo. Aristóteles (384-322 aC) refería que el conocimiento se obtiene a partir del pensamiento abstracto, el razonamiento y la comprensión o intelección(3); y que el mismo se obtiene a través de las vivencias, 
involucrando esferas cognitivas como memoria e imaginación siendo estas dominadas por la sensibilidad llegando así al conocimiento intelectual en donde se conceptualiza en conocimiento. Lo que se contrapone con Platón (429 -347 aC) en donde su posición era idealista abstracta.

Los empiristas ingleses de los siglos XVII y XVIII, en particular John Locke, George Berkeley y David Hume, sostenían que todo el conocimiento debía derivarse de ideas implantadas en la mente por medio de la percepción sensorial. Los positivistas tenían una visión algo más amplia y menos orientada hacia lo psicológico de lo que significan los hechos, pero compartían la opinión de los empiristas de que el conocimiento debía derivarse de los hechos de la experiencia. Los positivistas lógicos, una escuela filosófica que se originó en Viena en los años veinte de este siglo, retomó el positivismo introducido por Auguste Comte en el siglo XIX e intentó formalizarlo, prestando mucha atención a la forma lógica de la relación entre conocimiento científico y los hechos. (4)

\section{El Conocimiento}

En la actualidad para poder describir al conocimiento es necesario referir que la filosofía es la ciencia que estudia el pensamiento, siendo un intento del ser humano en llegar a la concepción del universo mediante la reflexión autónoma sobre las funciones y la valorización de las teorías y de las practicas, siendo así una reflexión sobre la conducta teórica a lo que llamaremos ciencia y la filosofía como la teoría del conocimiento científico o teoría de la ciencia.

Se puede definirse a la teoría del conocimiento como la teoría del pensamiento verdadero, a diferencia de la lógica que sería la teoría del pensamiento correcto. En el fenómeno del conocimiento se encuentran frente a frente la conciencia y el objeto: el sujeto y el objeto. Por ende, el conocimiento puede definirse como una determinación del sujeto por el objeto. Un conocimiento es verdadero si su contenido concuerda con el objeto mencionado. (5)

"La ciencia se deriva de los hechos", pero no se puede sostener gran parte de lo que comúnmente se supone que está implicado en dicho lema y los hechos son afirmaciones acerca del mundo que pueden ser verificadas directamente por un uso cuidadoso y desprejuiciado de los sentidos.

\section{El conocimiento en los sistemas organizados}

Es considerado como uno de los recursos más importantes de un sistema organizado por tener la capacidad de a partir de acciones tornar las decisiones, cuyas características organizacionales se reflejan en la expresión de la inteligencia, eficiencia y eficacia; estimulando la innovación manteniendo la complejidad, flexibilidad y creatividad.(6) este recurso intangible de las sociedades investigadoras aporta un relevante avance en cuanto a 
las decisiones que se toman a partir del producto final de las pesquisas realizadas a la población en general.

Históricamente, la responsabilidad del sistema médico (componente importante, pero reducido, del sistema salubrista) ha sido la de atender aquella porción de la población que acude a dicho sistema, es decir, la mortalidad y la morbilidad conocida.(7) Los sistemas públicos y privados de salud son organizaciones cuya misión es contribuir a mejorar la salud del individuo y de la sociedad (8) mediante la aplicación de protocolos previamente aprobados y establecidos por los entes regulatorios del sistema; los mismos que tienen que ser modificados y aprobados en las distintas poblaciones dando como resultado una atención más personalizada y relacionada con la realidad socio cultural de la población que se está atendiendo.

\section{Que es la Gestión del Conocimiento}

"La Gestión del conocimiento (GC) (del inglés Knowledge Management) es un concepto aplicado en las empresas, que pretende transferir el conocimiento y experiencia existente en los empleados, de modo que pueda ser utilizado como un recurso disponible para otros en la organización, el proceso requiere técnicas para capturar, organizar, almacenar el conocimiento de los trabajadores, para transformarlo en un activo intelectual que preste beneficios y se pueda compartir." (9)

La racionalidad hermenéutica lo refiere Habermas en la década de 1960 en donde existe una clara relación entre el conocimiento y el interés que guía el trabajo humano, discriminando el interés técnico, el interés práctico y el interés emancipatorio. (10)

Se percibe a la GC como sistema de recopilación, organización, análisis y divulgación de los conocimientos obtenidos dentro de un sistema organizativo, aportando satisfacciones en cuanto a la necesidad de crecimiento en cuanto al conocimiento; en si es el proceso mediante el cual una organización emplea su inteligencia colectiva para lograr sus objetivos estratégicos(11). Esto supone procesos que buscan la combinación sinergética de los datos y la capacidad de procesamiento de información de las tecnologías de la información y la comunicación y la capacidad creativa e innovadora de los seres humanos. La gestión de la información y el conocimiento surge como intento sistematizado de movilizar el capital humano y social de las organizaciones en función de la maximización de los beneficios de los sistemas organizados.

Gestión del Conocimiento en salud significa usar y crear conocimientos en una dinámica orientada a la solución de problemas de salud aprovechando la inteligencia colectiva y aportando a flujo global de conocimientos relacionado a la salud; puede enfocarse desde una dimensión que prioriza el empoderamiento de los individuos (capital humano) y comunidades (capital social) y el fortalecimiento del conocimiento científico-técnico (capital 
cultural); de manera que este se traduzca en políticas, programas y prácticas que puedan mejorar la calidad y esperanza de vida de las personas siendo así un proceso sistemático de encontrar, seleccionar, organizar, disponer, presentar y compartir información para transformarla mediante la práctica colaborativa en conocimientos.

Se trata de reconocer explícitamente un proceso que ha sido natural al desarrollo social pero que ahora cobra fuerza por las oportunidades que ofrecen los avances científicos técnicos y particularmente las tecnologías de la información y las comunicaciones con la digitalización $\mathrm{y}$ las redes.

El proceso de GC abarca toda la manera de generar, almacenar, distribuir y utilizar el conocimiento. Ese proceso envuelve el tratamiento de grandes volúmenes de datos, tornando necesaria la utilización de tecnologías de información para que presente eficiencia aceptable. Con el objetivo de promover el crecimiento, el desarrollo, la comunicación y la preservación del conocimiento dentro de una organización, la GC posibilita a que los profesionales alcancen respuestas rápidas y asertivas vinculadas a las decisiones que necesitan tomar.(12)

Es por ello que se propone que las políticas reconozcan el papel fundamental de la realidad que se pretende transformar y el papel predominante de los flujos locales de información y conocimiento asociados a los procesos de construcción de salud.

\section{¿Cómo se relaciona el conocimiento con la investigación en salud?}

Como lo refiere (11) "la investigación es una indagación sistemática y autocrítica y de inmediato pasa a relacionarse con la enseñanza" este complejo sistema de búsqueda tiene como objetivo obtener nuevo conocimiento manteniendo una racionalidad hermenéutica.

Una de las prioridades de Sistema Nacional de Salud, es la investigación (13), es una sistemática búsqueda de información y nuevos conocimientos; esta herramienta brinda dos esenciales y poderosas armas para los avances acelerados en salud, como la investigación básica o tradicional que es necesaria para generar nuevos conocimientos, tecnologías, y lograr mejores intervenciones acorde a los problemas de salud; y la investigación aplicada, es necesaria para el proceso de identificación y priorización de problemas así como para diseñar y evaluar políticas y programas que aportan al desarrollo científico, es un elemento clave para el éxito en la estrategia de año con el objetivo de mejorar la salud en la sociedad actual (14).

Para (15) refiere que es el parte del proceso aceptado y validado para solucionar interrogantes, encaminados a conocer los principios y leyes que sustentan al hombre y al mundo; posee sistemas de propios basados en el método de hipótesis deducción /inducción complementados con cálculos estadísticos y de probabilidades siendo así el estudio de rigurosidad científica de la respuesta social organizada y diseñada para enfrentar las 
condiciones de salud y enfermedad en las poblaciones para perfeccionar la eficiencia y eficacia del sistema de salud como parte integrante del proceso general de desarrollo socioeconómico, en la que sus miembros se interesan por el proceso, se ocupan de diseñar o de poner en práctica uno o más proyectos de investigación.(16)

La investigación en los sistemas de salud tiene como objetivo ayudar a los administradores de salud a lograr mayor utilidad y dinamismo de la gestión en salud, a través de la identificación de problemas prioritarios, la caracterización de los problemas, el análisis de las alternativas, la selección de estrategias de intervención y la evaluación de los resultados contribuyendo al progreso de la medicina al proporcionar las pruebas en que basar la práctica clínica y mejorar la calidad de la atención que se presta a los pacientes (17), obteniendo información sobre la utilidad y eficacia de los procedimientos diagnósticos, terapéuticos y preventivos, así como sobre la etiología, la fisiopatología y los factores de riesgo de las enfermedades y problemas de salud.

Siendo una irrefutable verdad que gran parte de los países desarrollados orientan sus servicios médicos a resolver los problemas curativos - paliativos. Pero los problemas de la población son mucho más amplios. Incluyen no sólo la parte visible del iceberg (aquellos pacientes que acuden al sistema) sino también su parte oculta.

La GC está compuesta por un grupo de procesos estratégicos que se producen en forma cíclica cuyos procesos internos son:

- Identificación del conocimiento

- Generación o Adquisición del conocimiento

- Desarrollo del Conocimiento

- Codificación o Transformación del Conocimiento

- Almacenamiento del Conocimiento

- Distribución o Compartir el Conocimiento

- Uso o Aplicación del Conocimiento

- Medición del Conocimiento

\section{El proceso investigativo}

Los progresos científicos y tecnológicos que día a día aportan el conocimiento de modernos procedimientos y avances en la medicina, por una parte, conllevan un sinnúmero de beneficios para las personas sanas o enfermas.(18)

La investigación científica parte de un proceso aceptado y validado para solucionar interrogantes, encaminados a conocer los principios y leyes que sustentan al hombre y mundo; posee sistemas de propios basados en el método de hipótesis deducción /inducción complementados con cálculos estadísticos y de probabilidades.(15) 
Los elementos básicos de la investigación científica, que caracteriza registros de hechos (resultados numéricos, textos, imágenes y sonidos) la que inicia al proponer una hipótesis para ajustar con modelos matemáticos de comprobación y finalizar estableciendo conclusiones valederas y reproducibles. (19)

El buen manejo de la teoría del conocimiento en investigación científica permite respuestas correctas y técnicas y una hipótesis, razón por la que el investigador científico debería conocer su teoría y evolución. (15) Siendo el reflejo indicado y expresivo de la realidad que se formaliza de manera lingüística(20) que comúnmente son aceptados en la comunidad científica por permitir la validación de resultados en la investigación científica. Un set de datos de investigación constituye una representación sistemática parcial del objeto que está siendo investigado. La información científica refiere a las conclusiones obtenidas del análisis de datos y a los resultados de una investigación (19)

El proceso científico no queda terminado cuando la investigación concluye ni cuando se presenta en un congreso o seminario, sino hasta que se ha publicado en una revista científica.(21) como refiere Wolf et. all.... Compartir el conocimiento es lo que llevó a nuestra especie al rol dominante que hoy en día ocupa.(22) La ciencia se caracteriza entonces por ser del conocimiento público (23) Siendo los repositorios y las revistas científicas son otra posibilidad que tienen los autores para difundir sus conocimientos. (24)

\section{La construcción de conocimiento en el sector Salud}

El conocimiento reside en el contexto subjetivo de la acción de los actores de salud y se basa en la información de que estos disponen en un momento dado, reside en los actores y en los grupos y no en las colecciones de información.

A diferencia de la información, el conocimiento está embebido en las personas y la creación de conocimientos ocurre en el proceso de interacción social. Se puede decir que existe estrecha relación entre los dos procesos, pero la Gestión de Información se engloba en la GC que a su vez la utiliza para generar nuevos conocimientos.

La salud pública exige cada vez más una respuesta anticipada de los actores involucrados y un ciclo de creación de conocimiento-acción mucho más rápido a partir de los nuevos conocimientos creados.

Esto significa que aunque en salud son necesarias soluciones basadas en actividades de procesamiento de información, el énfasis en la GC parece estar más del lado de las capacidades de los individuos de actuar y resolver problemas con la información disponible y con la creación de flujos de información y comunicación altamente flexibles y cambiantes.

La salud pública exige cada vez más una respuesta anticipada de los actores involucrados y un ciclo de creación de conocimiento-acción mucho más rápido a partir de los nuevos 
conocimientos creados. Esto significa que aunque en salud son necesarias soluciones basadas en actividades de procesamiento de información, el énfasis en la GC parece estar más del lado de las capacidades de los individuos de actuar y resolver problemas con la información disponible y con la creación de flujos de información y comunicación altamente flexibles y cambiantes.

Una de las prioridades de Sistema Nacional de Salud, es la investigación (13), siendo esta una sistemática búsqueda de información y nuevos conocimientos; esta herramienta brinda dos esenciales y poderosas armas para los avances acelerados en salud, como la investigación básica o tradicional que es necesaria para generar nuevos conocimientos, tecnologías, y lograr mejores intervenciones acorde a los problemas de salud; y la investigación aplicada, es necesaria para el proceso de identificación y priorización de problemas así como para diseñar y evaluar políticas y programas que aportan al desarrollo científico, es un elemento clave para el éxito en la estrategia de año con el objetivo de mejorar la salud en la sociedad actual (14).

Para Ramírez et. all. (15) refiere que es el parte del proceso aceptado y validado para solucionar interrogantes, encaminados a conocer los principios y leyes que sustentan al hombre y al mundo; posee sistemas de propios basados en el método de hipótesis deducción /inducción complementados con cálculos estadísticos y de probabilidades siendo así el estudio de rigurosidad científica de la respuesta social organizada y diseñada para enfrentar las condiciones de salud y enfermedad en las poblaciones para perfeccionar la eficiencia y eficacia del sistema de salud como parte integrante del proceso general de desarrollo socioeconómico, en la que sus miembros se interesan por el proceso, se ocupan de diseñar o de poner en práctica uno o más proyectos de investigación.(16)

\section{El conocimiento en los sistemas organizados}

De acuerdo a lo manifestado por Salmador (2004)(17), las décadas anteriores se han caracterizado por la evolución acelerada desde el punto de vista científico, tecnológico y social, caracterizándose por el surgimiento de la llamada sociedad posindustrial, en la cual se reconoció la importancia del conocimiento. Por lo tanto, y según los aportes notables y visionarios de especialistas en el área tales como Arrow, Marshall, Penrose, Selznick, Simon, Knight, Hayek, Drucker, Machlup, Bueno y Bell, (citados por Salmador, 2004), en esa moderna perspectiva se valoran las organizaciones. Especialm

ente en la década de los años noventa, surge gran interés sobre la generación y administración del conocimiento como nuevo enfoque asociado a la Teoría de la Empresa. Derivado de la anterior aseveración, en años recientes especialistas como Drucker, Nonaka y Takeuchi, Davenport y Prusak (citados por Salmador, 2004:p.27), han apreciado el rol transcendental 
del conocimiento como factor clave de la competitividad y creación de valor en la economía de las organizaciones.

\section{Conclusiones.}

- El conocimiento en salud tiene la importancia en promover el crecimiento, el desarrollo, la comunicación y la preservación del conocimiento dentro de las instituciones, dando respuestas rápidas y asertivas en la tomada de decisiones en la práctica clínica, siendo la gestión del conocimiento un recurso estratégico para la vida de las instituciones de salud y de las personas que en ellas trabajan.

\section{Referencias bibliográficas.}

1. Knowledge O. Taxonomies of Organizational Knowledge. Knowl Creat Diffus Util. 2008 ;

2. Valhondo D, Diaz G. Gestión del conocimiento : del mito a la realidad. Díaz de Santos; 2010. 378 p.

3. Aristóteles. Tópicos. Vasa. 2008;

4. Bate SP, Robert G. Knowledge management and communities of practice in the private sector: lessons for modernizing the National Health Service in England and Wales. Public Adm [Internet]. 2002 Jan 1 [cited 2017 Dec 22];80(4):643-63. Available from: http://doi.wiley.com/10.1111/1467-9299.00322

5. Vargas-Mendoza JE. Teorias del Conocimiento [Internet]. Asociación Oaxaqueña de Psicología A.C. 2006 [cited 2018 Jun 27]. p. 1. Available from: http://www.conductitlan.net/conocimiento.ppt

6. Sousa Brito Rocha E, Nagliate P, Bis Furlan C, Rocha K, Trevizan MA, Costa Mendes IA. Gestión del conocimiento en salud: revisión sistemática de la literatura. Rev Latino-Am Enferm. 2012;20(2):1-9.

7. Navarro V. Concepto actual de salud pública. Salud Pública. 1997;49-54.

8. Lázaro P, Pozo F, Ricoy J. Una estrategia de investigación en el sistema nacional de salud: II. Investigación en servicios de salud. Med Clin. 1995;

9. Desarrollo AR-P y, 2016 undefined. Gestión del conocimiento en la educación universitaria. revistascientificas.una.py [Internet]. [cited 2018 Mar 12]; Available from: http://revistascientificas.una.py/index.php/RE/article/view/729 
10. Habermas J, Jiménez M, Ivars JF, Santos LM, Vidal Beneyto J. Conocimiento e interés. Taurus; 1990.

11. Nazim M, Mukherjee B. Knowledge management in libraries : concepts, tools and approaches. Chandos Publishing is an imprint of Elsevier; 2016.

12. Montani S, Bellazzi R. Supporting decisions in medical applications: the knowledge management perspective. Int J Med Inform. 2002 Dec;68(1-3):79-90.

13. Bernal-Delgado E, Peiró S, Sotoca R. Prioridades de investigación en servicios sanitarios en el Sistema Nacional de Salud. Una aproximación por consenso de expertos. Vol. 20, Gaceta Sanitaria. 2006. p. 287-94.

14. Ortiz AP, Calo WA, Suárez-Balseiro C, Maura-Sardo M, Suárez E. Bibliometric assessment of cancer research in Puerto Rico, 1903-2005. Rev Panam Salud Pública. 2009;25(4):353-61.

15. Ramírez A V. La teoría del conocimiento en investigación científica. Am Coll Occup Environ Med. 2009;70(3):217-24.

16. Bunge M. Paradigmas y Revoluciones en ciencia y técnica. El Basilisco Rev Filos ciencias humanas. 1983;15(1975).

17. Fathalla MF. Guia practica de investigación en salud de investigación. World Health Organization 2004, editor. Washington; 2008. 5,6.

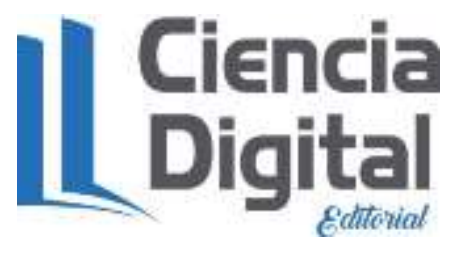




\section{PARA CITAR EL ARTÍCULO INDEXADO.}

Cañarte J., Marín C., Rivera L., Fernandez P. \& Huerta R. (2019). El conocimiento en el sistema de salud. Ciencia Digital 3(2), 508-518. Recuperado desde: http://cienciadigital.org/revistacienciadigital2/index.php/CienciaDigital/article/view/447/1011

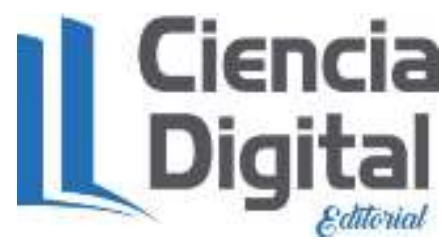

El artículo que se publica es de exclusiva responsabilidad de los autores y no necesariamente reflejan el pensamiento de la Revista Ciencia Digital.

El artículo queda en propiedad de la revista y, por tanto, su publicación parcial y/o total en otro medio tiene que ser autorizado por el director de la Revista Ciencia Digital.
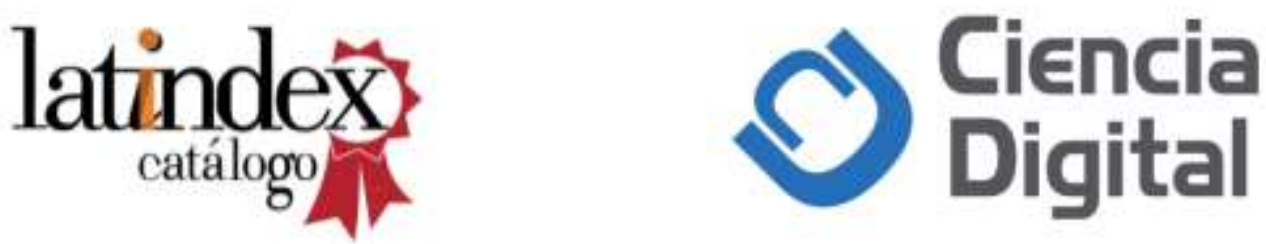